CASO CLÍNICO

\title{
Glomerulonefritis de rápida progresión pauciinmunitaria negativa a ANCA en un paciente de 18 años: a propósito de un caso
}

\author{
María N. Rivero*, M. Victoria Toledo, Carolina Díaz-Cuña y Sandra A. Consani \\ Servicio de Clínica Médica 3, Hospital Maciel, Montevideo, Uruguay
}

\section{Resumen}

La glomerulonefritis de rápida progresión (GNRP) pauciinmunitaria o de tipo III negativa a ANCA es infrecuente y representa tan sólo $10 \%$ a $20 \%$ del total de las GNRP pauciinmunitarias. Existen diferencias clínicas, analíticas y anatomopatológicas, entre las GNRP pauciinmunitarias negativas y positivas a ANCA. La sospecha de una GNRP pauciinmunitaria negativa a ANCA es elemental, ya que el pronóstico renal es tal vez peor en este subgrupo de pacientes. El tratamiento, al igual que en las GNRP de tipo III positivas a ANCA, se basa en la indicación de glucocorticoides y fármacos inmunosupresores.

Palabras clave: Glomerulonefritis pauciinmunitaria. Glomerulonefritis de rápida progresión. Vasculitis negativa a anticuerpo anticitoplasma de neutrófilos (ANCA). Insuficiencia renal aguda. Síndrome nefrótico.

\section{Rapidly progressive glomerulonefritis pauci-inmune ANCA negative, in an 18-year-old} patient, about a case

\section{Abstract}

Negative pauci-immune or type III ANCA GNRP represents only between $10 \%$ to $20 \%$ of total pauci-immune GNRP. There are clinical, analytical and pathological differences between the ANCA negative and positive pauci-immune GNRP. The suspicion of facing a negative ANCA pauci-immune GNRP is elementary, probably the renal prognosis is worse in this subgroup of patients. Treatment, as in ANCA positive type III GNRP, is based on the indication of glucocorticoids and immunosuppressive drugs.

Key words: Pauci-immune glomerulonephritis. Rapidly progressive glomerulonephritis. Anti-neutrophil cytoplasmic antibody (ANCA)-negative vasculitis. Acute renal failure. Nephrotic syndrome.

\section{Correspondencia:}

*María N. Rivero

E-mail: marianoelriv.28@gmail.com
Fecha de recepción: 30-07-2020

Fecha de aceptación: 27-04-2021

DOI: 10.24875/NEFRO.20000036
Disponible en internet: 18-06-2021

Nefro Latinoam. 2021;18:84-88 www.nefrologialatinoamericana.com 2444-9032/@ 2021 Sociedad Latinoamericana de Nefrología e Hipertensión. Publicado por Permanyer. Este es un artículo open access bajo la licencia CC BY-NC-ND (http://creativecommons.org/licenses/by-nc-nd/4.0/). 


\section{Puntos clave}

- Sólo 10\% a 20\% de las GNRP de tipo III son negativas a ANCA.

- El compromiso renal, establecido por la lesión glomerular, es más grave en este subgrupo de pacientes, en comparación con las GNRP de tipo III positivas a ANCA.

- El compromiso extrarrenal y los síntomas constitucionales se presentan en menor medida con respecto a las GNRP de tipo III positivas a ANCA.

- El tratamiento se basa en glucocorticoides $e$ inmunosupresores.

\section{Introducción}

La glomerulonefritis de rápida progresión (GNRP) define un síndrome clínico que se manifiesta por signos de daño glomerular (hematuria, proteinuria), junto con un deterioro gradual de la función renal con descenso de la filtración glomerular en más del 50\% o duplicación de la creatinina en un período de tiempo que puede comprender desde días hasta semanas ${ }^{1}$.

En términos histológicos se caracteriza por proliferación extracapilar glomerular y formación de semilunas. Estas últimas son la expresión morfológica de la proliferación de células parietales de la cápsula de Bowman, junto con la infiltración por monocitos circulantes que han atravesado la pared de los capilares glomerulares dañados y el depósito de fibrina ${ }^{2,3}$.

De manera característica, según sean los mecanismos etiopatogénicos, las GNRP se clasificaban en cuatro tipos: I, mediado por anticuerpos antimembrana basal glomerular; II, mediado por inmunocomplejos; III, sin depósito de inmunocomplejos o pauciinmunitario; y IV, combinación de características de los tipo I y III, con anticuerpos doblemente positivos.

La biopsia renal es la norma de referencia para el diagnóstico y las técnicas de inmunofluorescencia e inmunohistoquímica, junto con la microscopia electrónica, son esenciales para su tipificación ${ }^{2-4}$.

La GNRP pauciinmunitaria o de tipo III es la forma de presentación más frecuente en adultos mayores de 65 años, mientras que la GNRP de tipo II lo es en niños ${ }^{2}$. El diagnóstico y tratamiento tempranos son determinantes esenciales en su pronóstico².

A continuación se presenta un caso clínico peculiar de GNRP pauciinmunitaria en virtud de su negatividad para ANCA y la edad de presentación.

\section{Historia clínica}

Paciente femenino de 18 años, sin antecedentes personales de consideración ni familiares de enfermedad renal. Consulta por cuadro de hematuria franca total e indolora de 48 horas de evolución, acompañado de fiebre, astenia y adinamia, en el contexto de una infección respiratoria alta. Niega consumo de fármacos. La exploración física muestra a una paciente normotensa, apirética y sin taquicardia ni elementos indicativos de una enfermedad autoinmunitaria sistémica.

Las pruebas complementarias destacan hemoglobi-

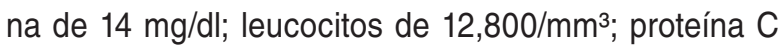
reactiva de $225 \mathrm{mg} / \mathrm{dl}$; urea plasmática de $0.60 \mathrm{mg} / \mathrm{dl}$ y creatinina plasmática de $3.11 \mathrm{mg} / \mathrm{dl}$, que ascienden hasta valores de $0.75 \mathrm{mg} / \mathrm{dl} \mathrm{y} 4.57 \mathrm{mg} / \mathrm{dl}$, respectivamente; albuminemia de $4.02 \mathrm{~g} / \mathrm{dl}$; complemento: C3 de $130 \mathrm{mg} / \mathrm{dl} \mathrm{y} \mathrm{C} 4$ de $37 \mathrm{mg} / \mathrm{dl}$. Examen general de orina: proteínas aisladas de $8.11 \mathrm{~g} / \mathrm{l}$, con aspecto turbio. $\mathrm{Ni}$ tritos positivos. Sedimento con abundantes eritrocitos y leucocitos. Urocultivo: $0 \mathrm{UFC} / \mathrm{ml}$. Índice proteína/ creatinina (pru/cru) de $3.5 \mathrm{~g} / \mathrm{g}$.

Se plantea la hospitalización con diagnóstico de insuficiencia renal grave de rápida evolución, con elementos de daño glomerular (proteinuria en límites nefróticos y hematuria), y glomerulonefritis aguda probablemente secundaria a enfermedad por IgA. Desde el punto de vista etiológico sobresalen complemento normal, ANA, ANCA, antimembrana basal glomerular, B-HCG, VDRL y virus de hepatitis $B$ y $C$ negativos. Para la detección de ANCA se realizó en primera instancia la técnica de inmunoanálisis con ELISA, con resultados negativos para los anticuerpos antiproteinasa 3 (anti-PR3) y antimieloperoxidasa (anti-MPO). En segunda instancia se aplicó la técnica de inmunofluorescencia indirecta (IFI), que arrojó también resultado negativo. Punción de biopsia renal (PBR): muestra renal cortical con 112 glomérulos. Siete glomérulos revelaron aumento de la celularidad a expensa de proliferación extracapilar celular; las semilunas son celulares y ocupan el $50 \%$ o más de la circunferencia glomerular. Se identifica necrosis glomerular. No se reconocen aumento de la celularidad: mesangial o endocapilar. Las membranas basales capilares viables son delgadas y regulares. El intersticio muestra fibrosis del $15 \%$ y escaso infiltrado inflamatorio linfomonocitario. Los túbulos muestran revestimiento epitelial conservado, en un sector aplanado, y citoplasma de aspecto microgranular; los túbulos proximales poseen ribete en cepillo parcialmente conservado. Hay cilindros tubulares hialinos aislados y cilindros de detritos 
y eritrocitarios. Las arteriolas y arterias muestran morfología conservada.

El estudio inmunopatológico de muestra renal cortical revela tres glomérulos, sin observar depósitos de $\lg \mathrm{A}, \lg \mathrm{G}, \operatorname{Ig} \mathrm{M}, \mathrm{C} 1 \mathrm{q}, \mathrm{C} 3$, kappa, lambda y fibrinógeno.

En suma, se establece el diagnóstico de glomerulonefritis proliferativa difusa extracapilar pauciinmunitaria, con proliferación extracapilar hasta de $58 \%$, con daño tubular agudo y sin evidencia de cronicidad. Arteriolas y arterias conservadas. Nota: el cuadro morfológico y el perfil de inmunofluorescencia sugieren vasculitis pauciinmunitaria (Fig. 1 y 2).

Se confirma entonces el diagnóstico de GNRP pauciinmunitaria de tipo III. Se instituye tratamiento de inducción con bolos de metilprednisolona (1 g día por tres días), seguido de prednisona (60 mg VO en el primer mes), con descenso progresivo, y ciclofosfamida ( $1 \mathrm{~g}$ IV mensual) por seis meses.

En la evolución se observa franca mejoría de la función renal y se registran al alta valores de urea plasmática de $0.56 \mathrm{mg} / \mathrm{dl}$ y creatinina plasmática de $0.75 \mathrm{mg} / \mathrm{dl}$, con índice pru/cru de $0.11 \mathrm{~g} / \mathrm{g}$.

\section{Discusión y conclusiones}

La GNRP pauciinmunitaria o de tipo III es el subtipo de GNRP que se presenta con mayor frecuencia en la población adulta y se caracteriza por la ausencia de depósitos de inmunocomplejos².

La gran mayoría de los casos de GNRP de tipo III se presenta con anticuerpos anticitoplasma de neutrófilo (ANCA) positivos (80\%-90\%) ${ }^{4}$. En el caso clínico analizado se destacan tres particularidades. En primer lugar, su forma de presentación clínica con hematuria en el contexto de una infección respiratoria, que orienta hacia una GNRP de tipo II por IgA. La anatomía patológica descartó esa posibilidad dado que se confirmó una GNRP de tipo III. En segundo lugar se reconoció la ausencia de ANCA en la forma histológica pauciinmunitaria, un hallazgo que ocurre en una baja frecuencia como se ha mencionado ya (10\%-20\%). En tercer lugar, la edad inhabitual de presentación.

El porcentaje de GNRP pauciinmunitaria negativa a ANCA se redujo con el paso de los años; hoy en día se acerca al $10 \%$. La disminución de este porcentaje se basa en el desarrollo de técnicas diagnósticas (inmunofluorescencia, inmunohistoquímica y microscopia electrónica), junto con la unificación de conceptos con respecto a la clasificación e informes de biopsias renales, establecido en el consenso de la Sociedad de Patología Renal en 2015 en la Clínica Mayo4. El

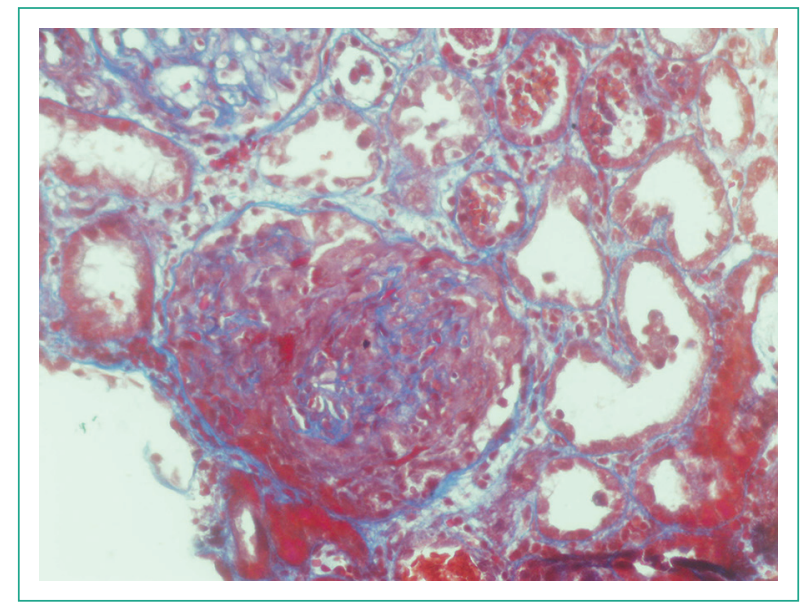

Figura 1. Glomérulo con técnica de tricrómico con semiluna circunferencial y necrosis.

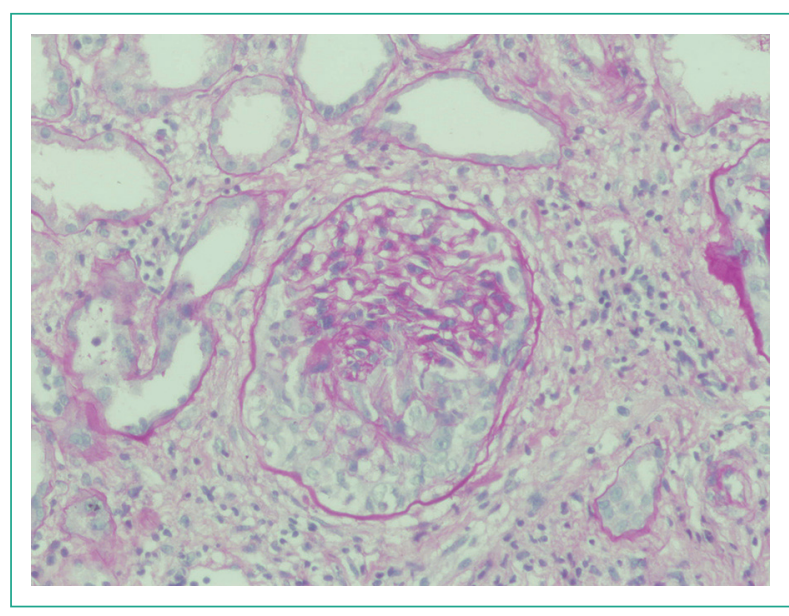

Figura 2. Semilunas celulares con ocupación del 50\% de la circunferencia.

objetivo de dicho consenso fue estandarizar la clasificación etiopatogénica junto al informe o lectura de biopsias renales en las glomerulonefritis (GN). Este grupo de investigadores clasificó las GN en cinco grupos: antimembrana basal glomerular, inmunocomplejos, pauciinmunitaria, GN Ig monoclonal y GN C3 ${ }^{4}$.

La glomerulonefritis C3 debe considerarse como diagnóstico diferencial sobre todo en pacientes jóvenes, si bien la clínica del caso presentado podría corresponder a una GN C3, la complementemia se halla en límites de normalidad y la inmunofluorescencia C3 negativa excluye ese diagnóstico.

En las GNRP de tipo III positivas a ANCA los dos antígenos principales, considerados los marcadores serológicos de las vasculitis ANCA relacionadas, son 
la proteinasa 3 (PR3) y la mieloperoxidasa (MPO). Se ha demostrado que la infusión de éstas no da lugar al desarrollo de glomerulonefritis en modelos animales. Con base en estos hallazgos, se ha propuesto que los ANCA no causan daño glomerular en forma directa, sino que los neutrófilos que ellos activan son los que producen los cambios histopatológicos ya descriptos mediante la liberación de agentes inflamatorios y proteínas de degradación, causantes del daño glomerular y la proliferación celular ${ }^{5}$.

La mayoría de los pacientes con GNRP pauciinmunitaria o de tipo III positiva a ANCA sufre compromiso glomerular en el contexto de vasculitis sistémicas de pequeño vaso, como la granulomatosis con poliangeítis, la poliangeítis microscópica y la granulomatosis eosinofílica con poliangeítis, aunque con menos frecuencia se limitan sólo al riñón².

Algunas investigaciones realizadas en Francia, Reino Unido, España y China se enfocaron en el estudio comparativo de dos grupos de pacientes: GNRP de tipo III positiva y negativa a ANCA.

Dos de estos estudios, uno conducido en Pekín de Min Chen, et al. ${ }^{6}$ y otro realizado en España de Villacorta, et al. ${ }^{7}$ concluyeron que la población de pacientes con GNRP de tipo III negativa a ANCA es significativamente más joven al momento del diagnóstico, en comparación con la población de pacientes con GNRP de tipo III positiva a ANCA, tal y como se observó en el caso clínico presentado ${ }^{5,6}$.

Existen otros hallazgos distintivos entre los pacientes con GNRP positivas y negativas a ANCA; en estas últimas se halló una mayor proteinuria en 24 horas, mayor proliferación mesangial ${ }^{7}$ y menor calificación BVAS (Birmingham vasculitis activity score) ${ }^{6}$. No se identificaron diferencias en la sobrevida global de los pacientes en ambos estudios. Sin embargo, en lo que respecta a la evolución natural de la GNRP pauciinmunitaria, el estudio en Pekín muestra peor sobrevida renal de los pacientes negativos a ANCA. En contraste, el estudio español no revela diferencias en la función renal ni en la necesidad de diálisis en ambos grupos $^{5,6}$.

Los mismos estudios establecen que el compromiso extrarrenal (pulmonar, gastrointestinal, oftalmológico, entre otros) y los síntomas constitucionales (fiebre, fatiga y pérdida de peso) se presentan en menor proporción en las GNRP III negativas a ANCA y se relacionan con anticuerpos ANCA. Sin embargo, en el plano renal se observa mayor lesión glomerular, lo que determina un mayor grado de proteinuria, como en el caso clínico presentado.
Se desconoce en la actualidad la etiopatogenia de la GNRP III negativa a ANCA. Se cree que la activación de neutrófilos por medios distintos respecto de los ANCA constituye el factor determinante. Los anticuerpos contra células endoteliales y proteína humana lisosomal 2 son algunos de los factores que intervienen en su activación?.

Sethi, et al. publicaron un estudio retrospectivo en el que se comparó la PBR de pacientes con GNRP pauciinmunitaria positiva y negativa a ANCA. Dichos autores observaron, en las muestras histológicas de pacientes negativos a ANCA, una mayor activación de neutrófilos y mayor acumulación de factores del complemento sobre todo de la vía alterna y final (C3, C9) del complemento. De acuerdo con esa observación concluyeron que el daño tisular local superior en la GNPR pauciinmunitaria negativa a ANCA podría relacionarse con alteraciones congénitas 0 adquiridas en la vía alterna del complemento ${ }^{8}$.

A pesar de representar las GNRP pauciinmunitarias negativas a ANCA un porcentaje pequeño de las GNRP de tipo III, y no conocer aún su etiología, se han descrito en fecha reciente casos en contextos de situaciones clínicas específicas, entre ellas las siguientes: el consumo de cocaína vinculado con levamisol en un paciente de 38 años $^{9}$, la utilización de nidostaurina (fármaco quimioterápico) en un paciente de 61 años portador de leucemia mieloide aguda ${ }^{10}$, un caso de un paciente de 64 años con diagnóstico de cáncer de pulmón microcítico y otros ejemplos relacionados con dicha malformación oncológica ${ }^{11}$ y un último caso vinculado con enfermedad de tejido mixto conectivo ${ }^{12}$.

El tratamiento no varía con respecto al de las GNRP de tipo III positivas a ANCA. Se basa en la indicación de bolos de metilprednisolona y fármacos inmunosupresores como la ciclofosfamida. Algunos de los estudios demuestran que el tratamiento es más efectivo en los pacientes portadores de ANCA, si bien esta afirmación no se sustenta en todos los estudios? ${ }^{2}$.

Se ha destacado que en todos los casos clínicos ya mencionados de GNRP pauciinmunitaria negativa a ANCA se instituyó el mismo tratamiento médico: bolos de metilprednisolona y ciclos de ciclofosfamida, seguidos de prednisona oral, todos con buena respuesta clínica y paraclínica ${ }^{9-12}$.

\section{Agradecimientos}

Los autores agradecen a la Dra. María Haydee Auchayna por proporcionar los materiales fotográficos de la punción de biopsia renal. 


\section{Financiamiento}

Esta investigación no ha recibido ninguna beca específica de agencias de los sectores públicos comercial o sin ánimo de lucro.

\section{Conflicto de intereses}

Los autores declaran no tener ningún conflicto de intereses.

\section{Responsabilidades éticas}

Protección de personas y animales. Los autores declaran que para esta investigación no se han realizado experimentos en seres humanos ni en animales.

Confidencialidad de los datos. Los autores declaran que han seguido los protocolos de su centro de trabajo sobre la publicación de datos de pacientes.

Derecho a la privacidad y consentimiento informado. Los autores han obtenido el consentimiento informado de los pacientes y/o sujetos referidos en el artículo. Este documento obra en poder del autor de correspondencia.

\section{Bibliografía}

1. Bielsa-Gracia S, Garzarán A, Del Agua C, Gascón A, Ruiz JE, Berisa F. Glomerulonefritis rápidamente progresiva a propósito de dos casos. $\mathrm{Ne}$ froplus 2016;8(2):170-178.
2. Chen M, Kallenberg C, Zhao M. ANCA-pauci-inmune crescentic glomerulonephiritis. Rev. Nephrol 2009;5:313-318.

3. Rahul M, Singh P, Misra V, Singh M, Mehrotra R, Tewarson S, et al. Comparative changes noted in renal biopsies on light microscopy of ANCA positive vs ANCA negative serology. J Clin Diagn Res. 2015;9(4):1-6

4. Sethi S, Hass M. Mayo clinic/renal pathology society consensus report on pathologic classification. Diagnosis and Reportin of GN. J Am soc Nephrol 2015;27:1-10.

5. Nakada $\mathrm{Y}$, Tsuboi N, Takahasi $\mathrm{Y}$, Yoshida $\mathrm{H}$, Hara $\mathrm{Y}$, Okonogi $\mathrm{H}$, et al A Case of concurrent MPO-/PR3-Negative ANCA-associated glomerulonephritis and membranous glomerulopathy. Case Repor Nephrol. 2015;2015:316863-8

6. Chen M, Yu F, Wang S, Zou W, Zhao W, Zhao M, et al. Antineutrophil cytoplasmic autoantibody-negative pauci-inmune crescentic glomerulonephritis. J Am Soc Nephrol. 2007:18:599-605.

7. Sharma A, Ritambra N, Naidu G, Minz R, Kohli H, Sarhuja V, et al. Pauci-immune glomerulonephritis: does negativity of antineutrophilic cytoplasmic antibodies matters? International Journal of Rheumatic Diseases. 2016;19:74-81.

8. Sethi S, Zand L, De Vriese A, Specks U, A. Vrana J, Kanwar S, et al. Comeplement activation in pauci-inmune necrotizing and crescentic glomerulonephritis: results of proteomic analysis. Nephrol Dial transplant. 2017;32:i139-i145.

9. Pimentel PVS, Freitas HC, Leite MDB, Lima RSA, Barreto DMS, Teixeira $A C$, et al. Rapidly progressive glomerulonephritis and acute kidney injury associated with cocaine use - case report. J Bras Nefrol. 2020:1-5. doi: 10.1590/2175-8239-JBN-2020-0034.

10. Pankow JD, -Carpentier R, Daver NG, Glass WF, Kala J. Unique case of ANCA-negative pauci-immune necrotizing glomerulonephritis with diffuse alveolar hemorrhage, potentially associated with midostaurin. CEN Case Rep. 2020 May;9(2):147-151. doi: 10.1007/s13730-020-00443-3. Epub 2020 Jan 18. PMID: 31955389; PMCID: PMC7148408.

11. Kancharla P, Surapaneni BK, Goldfinger M, Hennrick K, Ozeri DJ. Paraneoplastic seronegative pauci-immune glomerulonephritis associated with lung adenocarcinoma responds to rituximab: a case report. Case Rep Oncol. 2018 Jun 13:11(2):372-377. doi: 10.1159/000490101. PMID: 30022938; PMCID: PMC6047538.

12. Fernandes S, Teixeira C, Falcão LP, Costa AC, Raimundo M, Silva S, et al. ANCA negative pauci-immune crescentic glomerulonephritis and mixed connective tissue disease: a case study. J Bras Nefrol. 2019 Mar 18;42(2):245-249. doi: 10.1590/2175-8239-JBN-2019-0003. PMID: 30897193; PMCID: PMC7427652. 